\title{
Trajectory Prediction of Spin-Stabilized Projectiles with a Liquid Payload
}

\author{
G. R. Cooper* \\ U.S. Army Research Laboratory, Aberdeen Proving Ground, Maryland 21005-5069 \\ and \\ Mark Costello \\ Georgia Institute of Technology, Atlanta, Georgia 30332 \\ DOI: $\underline{10.2514 / 1.52564}$
}

\begin{abstract}
Payloads that behave like a liquid are carried onboard some projectile configurations, and it is well established that the internal motion of a liquid payload can induce destabilizing moments on the projectile. This paper creates a method to include the effect of a liquid payload in the flight dynamic equations of motion, enabling trajectory simulations of projectiles with liquid payloads. To include this effect, liquid payload moments are added to the applied loads on the projectile. These loads are computed by solving the linearized Navier-Stokes equations for a projectile undergoing coning motion. To highlight the methodology, trajectory simulation results are provided for an example projectile with different liquid payloads configurations possessing stable behavior while one exhibits catastrophic flight instability.
\end{abstract}

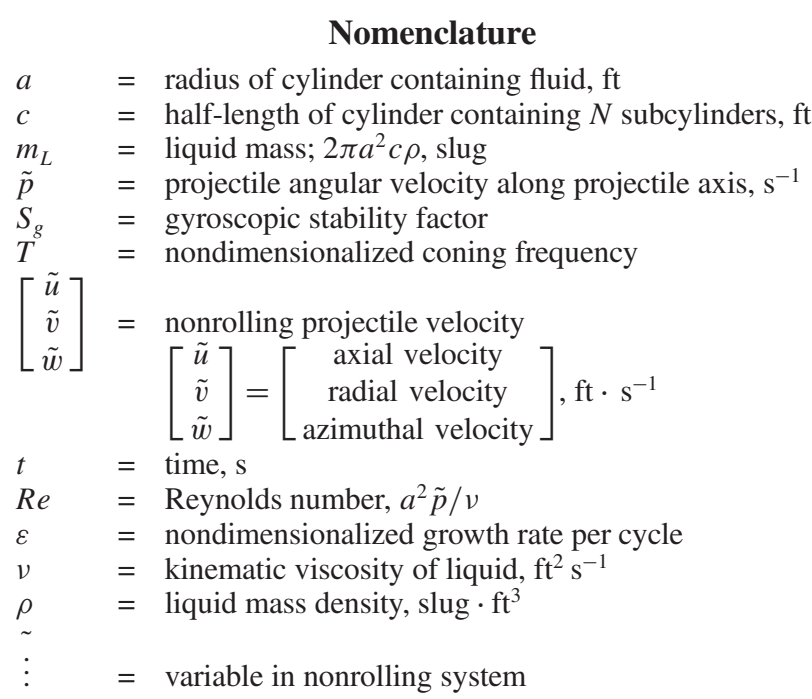

\section{Introduction}

W HILE the bulk of projectiles in use today behave as rigid bodies while in flight, a notable number of projectiles are purposefully designed to carry a liquid payload. For example, smoke screens delivered by artillery rounds consist of a typical spinstabilized shell containing a canister filled with white phosphorous [1-4]. Another example is new less-than-lethal projectiles having a concentric cylindrical cavity filled with liquid, which delivers this payload to a target upon impact [5]. Finally, some projectiles are designed to be general payload delivery shells, including delivery of medical supplies such as intravenous fluid bags [6].

There can be a significant difference in flight behavior between liquid-filled and solid-filled projectiles. The difference is caused by motion of the liquid inside the spinning projectile. This motion

Received 28 September 2010; revision received 1 February 2011; accepted for publication 4 February 2011. This material is declared a work of the U.S. Government and is not subject to copyright protection in the United States. Copies of this paper may be made for personal or internal use, on condition that the copier pay the $\$ 10.00$ per-copy fee to the Copyright Clearance Center, Inc., 222 Rosewood Drive, Danvers, MA 01923; include the code 0022-4650/ 11 and $\$ 10.00$ in correspondence with the CCC.

*Weapons and Materials Research Directorate. Senior Member AIAA.

${ }^{\dagger}$ Sikorsky Associate Professor, School of Aerospace Engineering. Associate Fellow AIAA. causes forces to act on the projectile body, which can prematurely terminate the flight by instability. Characteristics of this instability are sharp increases in angle of attack (AOA) accompanied by large changes in spin rate [7-10]. At gun launch, the motion of the projectile causes the fluid to spin up in a time-dependent manner, but it subsequently achieves steady flow.

Prediction of instability induced by a liquid payload installed in a coning projectile has been analyzed by computational fluid dynamic (CFD) theories and with analytic linear fluid theories based on spatial-eigenvalue methods. The CFD solutions are generally best suited to liquids with a low Reynolds number $R e$, while spatialeigenvalue methods can encompass a much broader range of Re. A good survey of liquid-filled projectiles with a focus on flight instabilities is given in [11]. Generally, analytic theories are a composition of inviscid flow and viscous boundary-layer corrections [12-14]. Prediction of flight stability of a liquid-filled projectile has also been studied using tricyclic linear-projectile theory [15]. This analysis assumes the effect of a liquid payload is similar to the Magnus effect. Spectral analysis used to numerically compute liquidfill-induced moments has been incorporated into six-degree-offreedom (6-DOF) simulations [16]. The angular motion of a low $R e$ liquid-filled projectile has also been simulated using a precomputed table of liquid-fill moments, obtained from CFD, in 6-DOF calculations [17].

The present paper meshes a well-developed spatial-eigenvalue theory directly into a standard 6-DOF projectile flight dynamic model. Hence, a well established rotating liquid model calculates liquid moments at each time step of the 6-DOF trajectory numerical simulation without the need of interpolation to gain the effects of liquid payloads. This paper further bridges the gap between the body of literature on effects of viscous liquid payloads on projectiles and 6-DOF projectile flight dynamic modeling. The paper begins with a review of rigid projectile flight dynamic modeling along with a description of modeling a rotating liquid in a cylindrical cavity. The two models are subsequently integrated such that a projectile flight dynamic model with a liquid payload results. To highlight the utility of the methodology, the flight dynamic model is exercised on an example shell. Comparisons are made between a liquid-filled projectile and a similar solid-filled projectile highlighting the predictive capability of the new model.

\section{Projectile Flight Dynamic Model with Liquid Payload}

A typical 6-DOF rigid projectile model is employed to predict the dynamics of a projectile in flight. These equations assume a flat 


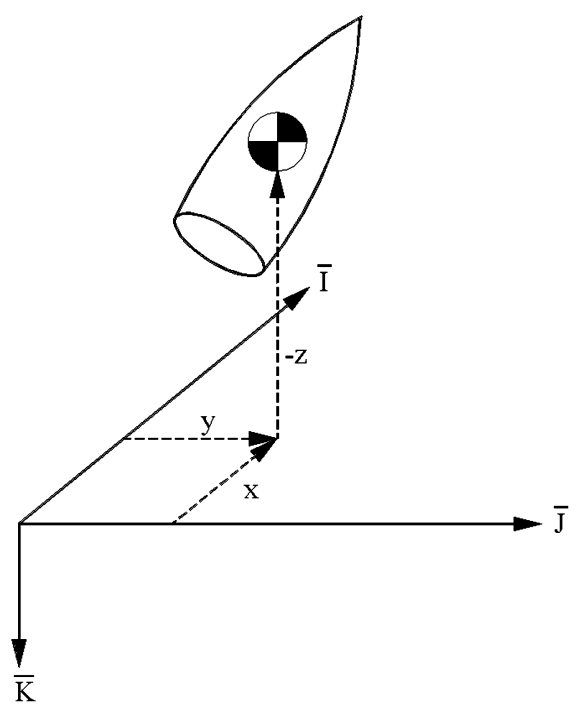

Fig. 1 Projectile position coordinate definitions.

Earth. The well-known 6-DOF states comprise the three translational components describing the position of the projectile's center of mass and the three Euler angles describing the orientation of the projectile with respect to the Earth. Figures $\underline{1}$ and $\underline{2}$ provide a visualization of the degrees of freedom.

The equations of motion [18] derived in the no-roll frame are shown in Eqs. (1- $\underline{4})$ :

$$
\begin{aligned}
& \left\{\begin{array}{c}
\dot{x} \\
\dot{y} \\
\dot{z}
\end{array}\right\}=\left[\begin{array}{ccc}
c_{\theta} c_{\psi} & -s_{\psi} & s_{\theta} c_{\psi} \\
c_{\theta} s_{\psi} & c_{\psi} & s_{\theta} s_{\psi} \\
-s_{\theta} & 0 & c_{\theta}
\end{array}\right]\left\{\begin{array}{c}
\tilde{u} \\
\tilde{v} \\
\tilde{w}
\end{array}\right\} \\
& \left\{\begin{array}{c}
\dot{\phi} \\
\dot{\theta} \\
\dot{\psi}
\end{array}\right\}=\left[\begin{array}{ccc}
1 & 0 & t_{\theta} \\
0 & 1 & 0 \\
0 & 0 & 1 / c_{\theta}
\end{array}\right]\left\{\begin{array}{l}
\tilde{p} \\
\tilde{q} \\
\tilde{r}
\end{array}\right\} \\
& \left\{\begin{array}{c}
\dot{\tilde{u}} \\
\dot{\tilde{v}} \\
\dot{\tilde{w}}
\end{array}\right\}=\left\{\begin{array}{c}
\tilde{X} / m \\
\tilde{Y} / m \\
\tilde{Z} / m
\end{array}\right\}-\left[\begin{array}{ccc}
0 & -\tilde{r} & \tilde{q} \\
\tilde{r} & 0 & \tilde{r} t_{\theta} \\
-\tilde{q} & -\tilde{r} t_{\theta} & 0
\end{array}\right]\left\{\begin{array}{c}
\tilde{u} \\
\tilde{v} \\
\tilde{w}
\end{array}\right\}
\end{aligned}
$$

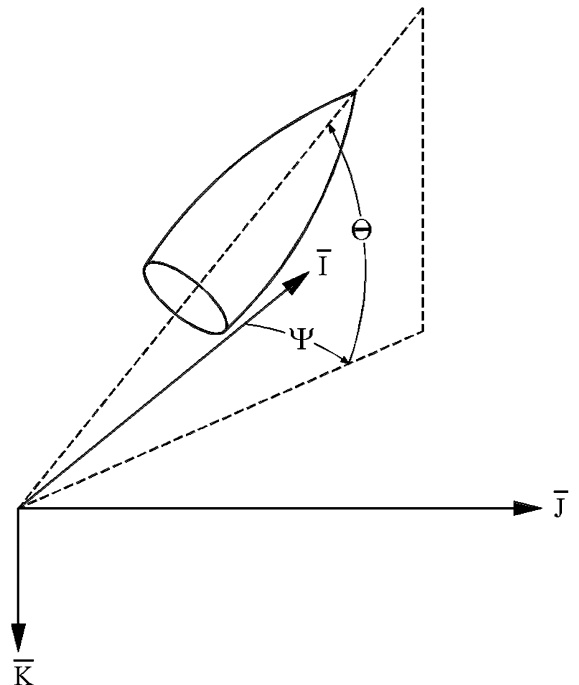

Fig. 2 Projectile orientation definitions.

$$
\left\{\begin{array}{c}
\dot{\tilde{p}} \\
\dot{\tilde{q}} \\
\tilde{r}
\end{array}\right\}=[I]^{-1}\left(\left\{\begin{array}{c}
\tilde{L} \\
\tilde{M} \\
\tilde{N}
\end{array}\right\}-\left[\begin{array}{ccc}
0 & -\tilde{r} & \tilde{q} \\
\tilde{r} & 0 & \tilde{r} t_{\theta} \\
-\tilde{q} & -\tilde{r} t_{\theta} & 0
\end{array}\right][I]\left\{\begin{array}{c}
\tilde{p} \\
\tilde{q} \\
\tilde{r}
\end{array}\right\}\right)
$$

Note that the following shorthand notation for trigonometric functions is used: $s_{\alpha}=\sin (\alpha), c_{\alpha}=\cos (\alpha)$, and $t_{\alpha}=\tan (\alpha)$ in Eqs. (1-4).

The force acting on the projectile in Eq. (3) comprises the weight force $W$ and the aerodynamic force. The aerodynamic force is split into standard $A$ and Magnus $M$ aerodynamic forces. The combination of forces is expressed in Eq. (ㅁ):

$$
\left\{\begin{array}{c}
\tilde{X} \\
\tilde{Y} \\
\tilde{Z}
\end{array}\right\}=\left\{\begin{array}{c}
\tilde{X}_{W} \\
\tilde{Y}_{W} \\
\tilde{Z}_{W}
\end{array}\right\}+\left\{\begin{array}{c}
\tilde{X}_{A} \\
\tilde{Y}_{A} \\
\tilde{Z}_{A}
\end{array}\right\}+\left\{\begin{array}{c}
\tilde{X}_{M} \\
\tilde{Y}_{M} \\
\tilde{Z}_{M}
\end{array}\right\}
$$

Equation (6) provides the expression for the weight force in the noroll coordinate system:

$$
\left\{\begin{array}{c}
\tilde{X}_{W} \\
\tilde{Y}_{W} \\
\tilde{Z}_{W}
\end{array}\right\}=m g\left\{\begin{array}{c}
-s_{\theta} \\
0 \\
c_{\theta}
\end{array}\right\}
$$

Equation (7) provides the expression for the aerodynamic force in the no-roll coordinate system. This force acts upon the projectile at the aerodynamic center of pressure:

$$
\left\{\begin{array}{c}
\tilde{X}_{A} \\
\tilde{Y}_{A} \\
\tilde{Z}_{A}
\end{array}\right\}=-\frac{\pi}{8} \rho V_{A}^{2} D^{2}\left\{\begin{array}{c}
C_{X 0}+C_{X 2}\left(\tilde{v}^{2}+\tilde{w}^{2}\right) / V_{A}^{2} \\
C_{Y 0}+C_{Y B 1} \tilde{v} / V_{A} \\
C_{Z 0}+C_{Z A 1} \tilde{w} / V_{A}
\end{array}\right\}
$$

Equation ( $\underline{8}$ ) provides the expression for the Magnus force in the noroll coordinate system. The Magnus force acts upon the projectile at the Magnus force center of pressure:

$$
\left\{\begin{array}{c}
\tilde{X}_{M} \\
\tilde{Y}_{M} \\
\tilde{Z}_{M}
\end{array}\right\}=\frac{\pi}{8} \rho V_{A}^{2} D^{2}\left\{\begin{array}{c}
0 \\
\frac{\tilde{p} D C_{N P A} \tilde{w}}{2 V_{A}^{2}} \\
\frac{-\tilde{p} D C_{N P A} \tilde{v}}{2 V_{A}^{2}}
\end{array}\right\}
$$

Equations (7) and (8) are based on Mach-number-dependent coefficients and the total aerodynamic velocity given in Eq. (9):

$$
V=\sqrt{\tilde{u}^{2}+\tilde{v}^{2}+\tilde{w}^{2}}
$$

The moment acting on the projectile in Eq. (4) comprises the moment due to the standard aerodynamic force $A$, the moment due to the Magnus aerodynamic force $M$, the unsteady aerodynamic moment $U A$, and the liquid payload moment $L$, as shown in Eq. (10):

$$
\left\{\begin{array}{c}
\tilde{L} \\
\tilde{M} \\
\tilde{N}
\end{array}\right\}=\left\{\begin{array}{c}
\tilde{L}_{A} \\
\tilde{M}_{A} \\
\tilde{N}_{A}
\end{array}\right\}+\left\{\begin{array}{c}
\tilde{L}_{M} \\
\tilde{M}_{M} \\
\tilde{N}_{M}
\end{array}\right\}+\left\{\begin{array}{c}
\tilde{L}_{U A} \\
\tilde{M}_{U A} \\
\tilde{N}_{U A}
\end{array}\right\}+\left\{\begin{array}{c}
\tilde{L}_{L} \\
\tilde{M}_{L} \\
\tilde{N}_{L}
\end{array}\right\}
$$

The moment due to the aerodynamic force is expressed in Eq. (11):

$$
\left\{\begin{array}{c}
\tilde{L}_{A} \\
\tilde{M}_{A} \\
\tilde{N}_{A}
\end{array}\right\}=\left[\begin{array}{ccc}
0 & -R_{C Z} & R_{C Y} \\
R_{C Z} & 0 & -R_{C X} \\
-R_{C Y} & R_{C X} & 0
\end{array}\right]\left\{\begin{array}{c}
\tilde{X}_{A} \\
\tilde{Y}_{A} \\
\tilde{Z}_{A}
\end{array}\right\}
$$

The moment due to the Magnus force is expressed in Eq. (12):

$$
\left\{\begin{array}{c}
\tilde{L}_{M} \\
\tilde{M}_{M} \\
\tilde{N}_{M}
\end{array}\right\}=\left[\begin{array}{ccc}
0 & -R_{M Z} & R_{M Y} \\
R_{M Z} & 0 & -R_{M X} \\
-R_{M Y} & R_{M X} & 0
\end{array}\right]\left\{\begin{array}{c}
\tilde{X}_{M} \\
\tilde{Y}_{M} \\
\tilde{Z}_{M}
\end{array}\right\}
$$

The unsteady aerodynamic moments acting on the projectile are expressed in Eq. (13): 


$$
\left\{\begin{array}{c}
\tilde{L}_{U A} \\
\tilde{M}_{U A} \\
\tilde{N}_{U A}
\end{array}\right\}=\frac{\pi}{8} \rho V_{A}^{2} D^{3}\left\{\begin{array}{c}
C_{D D}+\frac{\tilde{p} D C_{L P}}{2 V_{A}} \\
\frac{\tilde{q} D C_{M Q}}{2 V_{A}} \\
\frac{\tilde{r} D C_{N R}}{2 V_{A}}
\end{array}\right\}
$$

The coefficients used in this model are specific functions of the Mach number of the projectile. For fin-stabilized projectiles, Magnus force and moment are typically ignored, since their effect is rather small for slowly rolling projectiles.

The angular motion of a projectile is altered by the inertial waves propagating through the liquid payload. In turn, these waves act on the walls of the liquid cavity and generate a liquid moment. The impact of this moment can have a devastating impact on the projectile's angular motion [19-21]. For this paper, the liquid moment is calculated from steady linear-projectile theory, which incorporates solutions of the linearized Navier-Stokes equations [18]. In general, this liquid moment model is a linear combination of fast- and a slow-mode liquid moment contributions. At the top of page 16 of [19], Murphy states that, for a steady-state coning projectile with a liquid payload, ".....only the fast-mode motion is adversely affected by the liquid side moment." For this reason, only the fast coning mode is used to calculate the liquid moment in this paper. In particular, the liquid transverse and roll liquid moments have the following form:

$$
\begin{gathered}
{\left[\begin{array}{c}
\tilde{L}_{L} \\
\tilde{M}_{L} \\
\tilde{N}_{L}
\end{array}\right]=m_{L} a^{2} \tilde{p}^{2} T\left[\begin{array}{ccc}
1 & 0 & 0 \\
0 & c_{\beta} & s_{\beta} \\
0 & -s_{\beta} & c_{\beta}
\end{array}\right]\left[\begin{array}{c}
K^{2} C_{L R M} \\
K C_{L S M} \\
K C_{L I M}
\end{array}\right]} \\
C_{L R M}=-C_{L S M}+\frac{T \varepsilon}{2}\left[1-\frac{4}{3}\left(\frac{c}{a}\right)^{2}\right] \\
K=\frac{\sqrt{\tilde{v}^{2}+\tilde{w}^{2}}}{V}, \quad \beta=\frac{\tilde{v}}{\tilde{w}}
\end{gathered}
$$

The moment coefficients $C_{L S M}$ and $C_{L I M}$ depend on the fast-mode coning frequency $T$, fast-mode undamping $\varepsilon$, cavity aspect ratio $c / a$, liquid Reynolds number $R e$, and magnitude of axial spin [19]. Values of the fast-mode pair $(T, \varepsilon)$ are obtained from Eq. $(\underline{15})$ :

$$
\begin{aligned}
T & =\frac{\sqrt{2 \tilde{p}^{2}\left(I_{X}+a^{2} m_{L} C_{L I M}\right)^{2}-\pi \rho C_{N A} D^{2} I_{Y} R_{C X} V^{2}}}{2 \sqrt{2} \tilde{p} I_{Y}} \\
& +\frac{I_{X}+a^{2} m_{L} C_{L I M}}{2 I_{Y}} \\
\varepsilon & =\frac{\pi \rho D^{2}\left(D\left(C_{M Q} T+C_{Y P A} R_{M X}\right) / 2-C_{N A}\left(I_{Y} T-I_{X}\right) / M\right) V}{8 \tilde{p} T\left(2 I_{Y} T-I_{X}\right)} \\
& +\frac{a^{2} m_{L} C_{L S M}}{\left(2 I_{Y} T-I_{X}\right)}
\end{aligned}
$$

and the liquid moment coefficients $C_{L S M}$ and $C_{L I M}$ are calculated using the techniques given by Murphy [19].

\section{Results}

The example simulations given here consider liquid payloads in a typical projectile with mass properties $I x=0.1157 \mathrm{slug} \cdot \mathrm{ft}^{2}$, $I y=I z=1.1972 \mathrm{slug} \cdot \mathrm{ft}^{2}$, projectile weight $=103.0 \mathrm{lbf}$, and projectile diameter $=0.510 \mathrm{ft}$. At launch, the projectile has velocity $V=2460.0 \mathrm{ft} \cdot \mathrm{s}^{-1}$ and axial spin rate $\tilde{p}=1500.0 \mathrm{~s}^{-1}$. The liquid density $\rho_{L}$ is taken to be 3.5 times the density of liquid water, and the viscosity $v$ is selected such that launch $R e \geq 8 \times 10^{6}$. This is sufficient to ensure the Reynolds number remains large so boundarylayer analysis [18] adequately governs the liquid physics for the entire trajectory. The liquid cavity is a cylinder, with aspect ratio $c / a=3.75$, completely filled with this low-viscosity liquid. The range of nondimensional coning frequencies $T$ for a typical trajectory of this projectile housing a frozen (solid) liquid payload is given in Fig. 3 .

The Reynolds number for a given aspect ratio can cause large variations in the liquid moment when subjected to a range of nondimensional coning frequencies $T$. Applying steady-state linear liquid theory to a payload configuration undergoing coning motion reveals, in Fig. $\underline{4}$, the side moment coefficient $C_{L S M}$ with $c / a=3.75$, for two numbers: $R e=8 \times 10^{6}$ and $8 \times 10^{8}$. These results depict important $C_{L S M}$ behavior, where the peaks indicate a potential problem due to large liquid moments when the nondimensional coning rate $T \approx 0.088$. Note that the amplitude of the peak has a

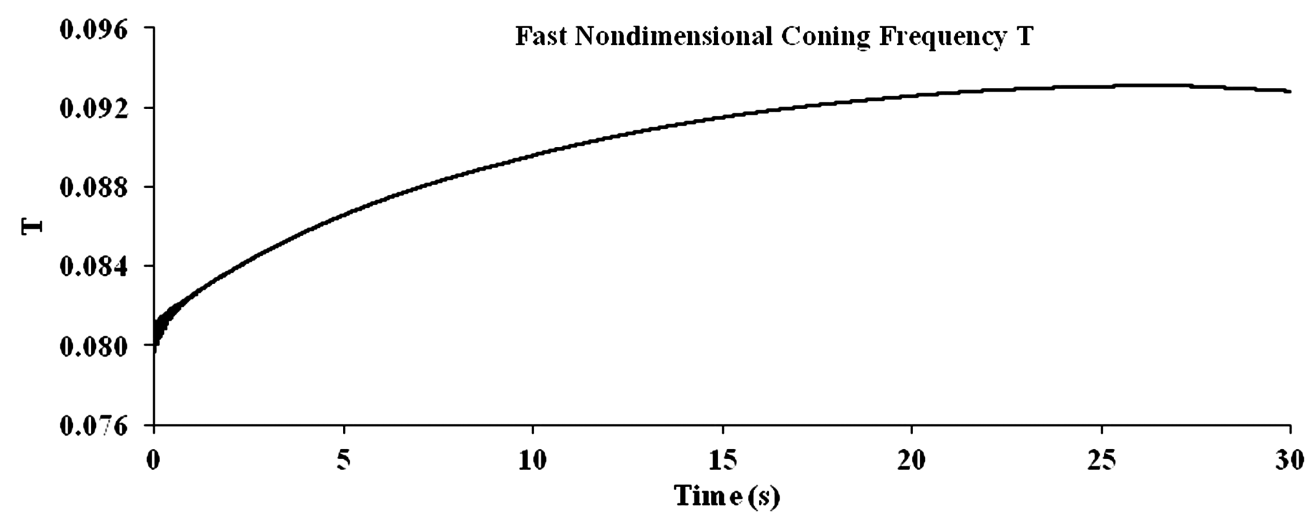

Fig. 3 Nondimensional fast-mode coning frequencies.

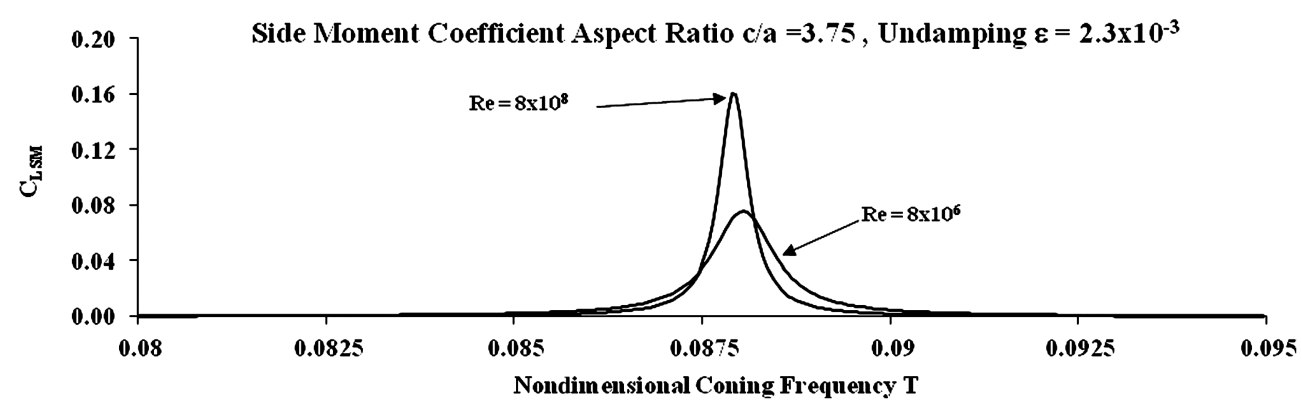

Fig. $4 C_{L S M}$ vs coning frequency and $R e$; resonance $T \approx 0.088$. 
strong dependence on $R e$. Peak values of $C_{L S M}$ are close to two orders of magnitude, larger than the nonpeak values of $C_{L S M}$.

The chart in Fig. 4 suggests, for a sufficiently large $R e$, a free flight missile having nondimensional coning frequencies in the neighborhood of $T=0.088$ will experience a significantly larger liquid moment than it does for frequencies outside this neighborhood. Results such as these are characteristic of the root cause of projectile instabilities due to liquid payloads. The aspect ratio coupled with a large enough Reynolds number forces inertial waves in the coning fluid to generate large $C_{L S M}$ coefficients [19]. In general, increasing $R e$ causes the liquid side moment to increase near $T=0.088$, and this pronounced peak signifies a possible resonant frequency. This is one indicator of a liquid payload possibly causing the fast coning frequencies to change such that an instability occurs near $T_{1}=.088$. Note that the range of frequencies given in Fig. 4 is close to the fastmode $T$ frequencies for the frozen liquid found in Fig. 3 .

Next, we present a series of results showing the effect a liquid payload has on projectile angular motion during flight. Results presented here are for a launch $R e=8 \times 10^{6}$, which is large enough so a linear liquid theory with boundary-layer corrections is valid throughout the entire trajectory. Figures 5 and 6 compare roll moment and spin rate results for a frozen liquid and a flowing liquid. These variables are selected, since the rotational physics of a projectile is a strong indicator of flight instability caused by liquid payloads [18].

The liquid payloads for the launch $R e$ chosen in these examples also have a small effect on projectile roll rate.
Evidently, the increased magnitude of the projectile roll moment due to the flowing liquid is not sufficient to cause flight instability.

The liquid side moment coefficient $C_{L S M}$ is an important parameter when evaluating the impact a liquid payload has on projectile angular motion. Figure 7 shows the fast-mode liquid moment coefficient as a function of $\bar{T}$.

Figure $\underline{8}$ compares the AOA for identical projectiles with payloads supporting inertial waves and frozen liquid payloads.

Apparently, the inertial wave motion slightly increases the AOA for a liquid with launch $R e=8.0 \times 10^{6}$ and $8.0 \times 10^{7}$, but this increase is not enough to cause concern of flight instability. In fact, the results presented so far show no indication of flight instability, even when the projectile has a coning rate near $T_{1}=0.088$ for the potential problem in $C_{L S M}$, found in Figs. 4 and 7 .

To understand the dynamics of a liquid-filled projectile exhibiting angular instability, we consider Fig. 4 and exploit the peak value of $C_{L S M}$ near $T=0.088$. Thus, to substantially increase the size of the liquid moment, we assume the payload comprises a hypothetical liquid with a decreased viscosity, so that the launch Reynolds number is large enough to cause substantial increases in liquid side moment during flight. In particular, we want this liquid to generate large enough values of $C_{L S M}$ for $T \approx 0.088$ to alter the angular motion of the projectile. A sufficiently large decrease in projectile spin rate, along with a large increase in AOA, suggests the hypothetical liquid may cause premature termination of flight. Figure 9 has a comparison showing the fast nondimensional $T$ dependence of the side moment coefficient $C_{L S M}$ rapidly increasing near $T=0.088$ for launch

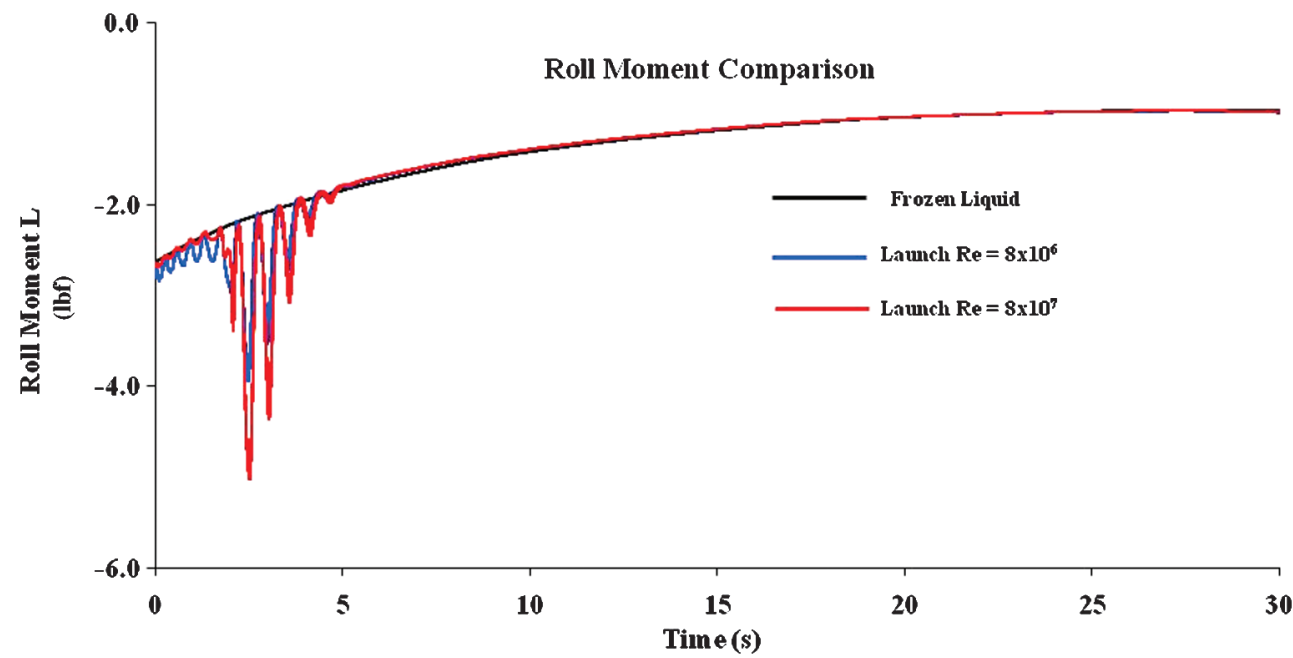

Fig. 5 Projectile roll moment for frozen and flowing liquid payloads.

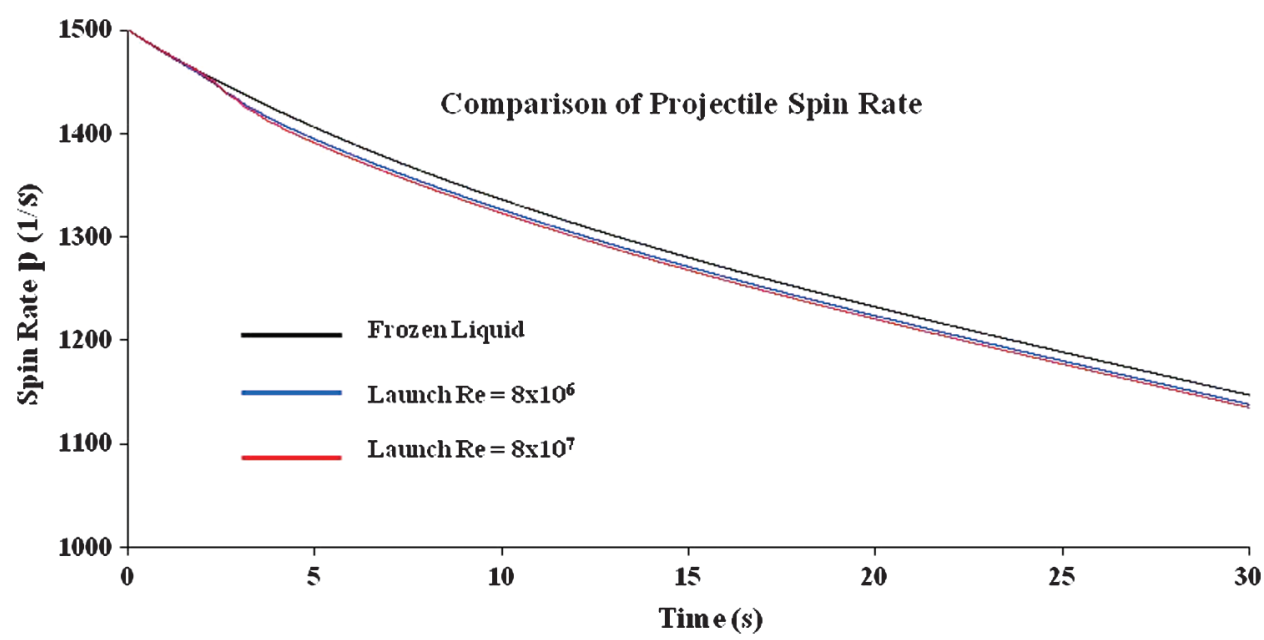

Fig. 6 Projectile spin rate for frozen and flowing liquid payloads. 


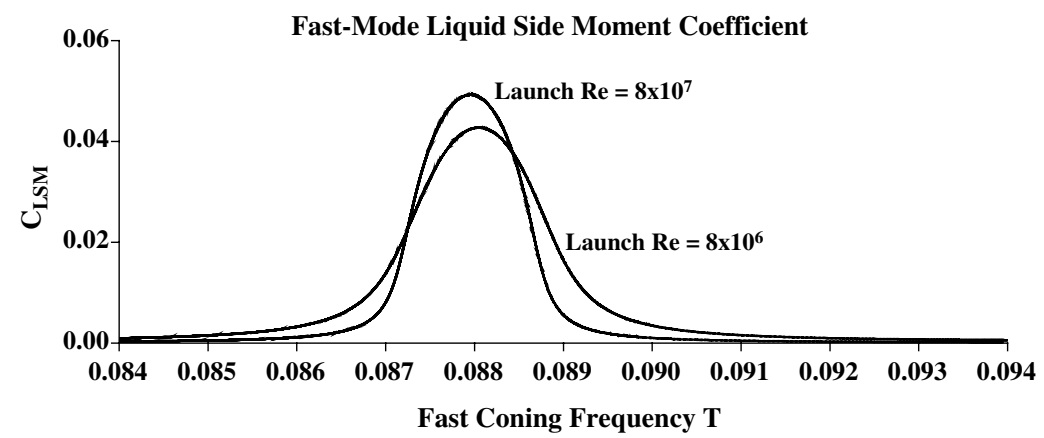

Fig. 7 Liquid moment coefficients vs nondimensional fast-mode frequency.

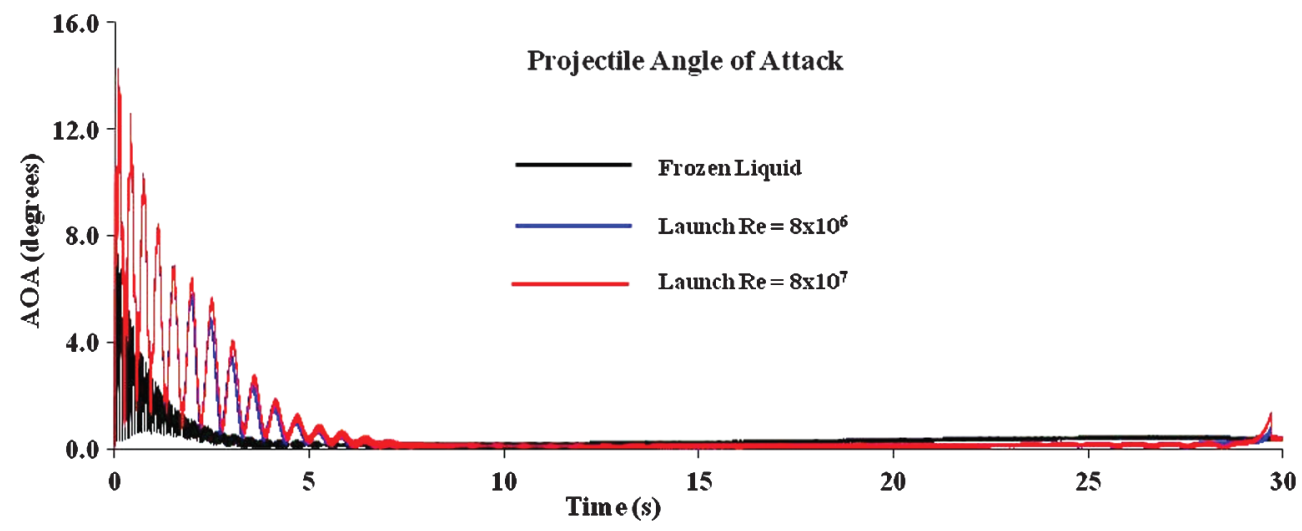

Fig. 8 Comparison of AOA of frozen and liquid payloads.

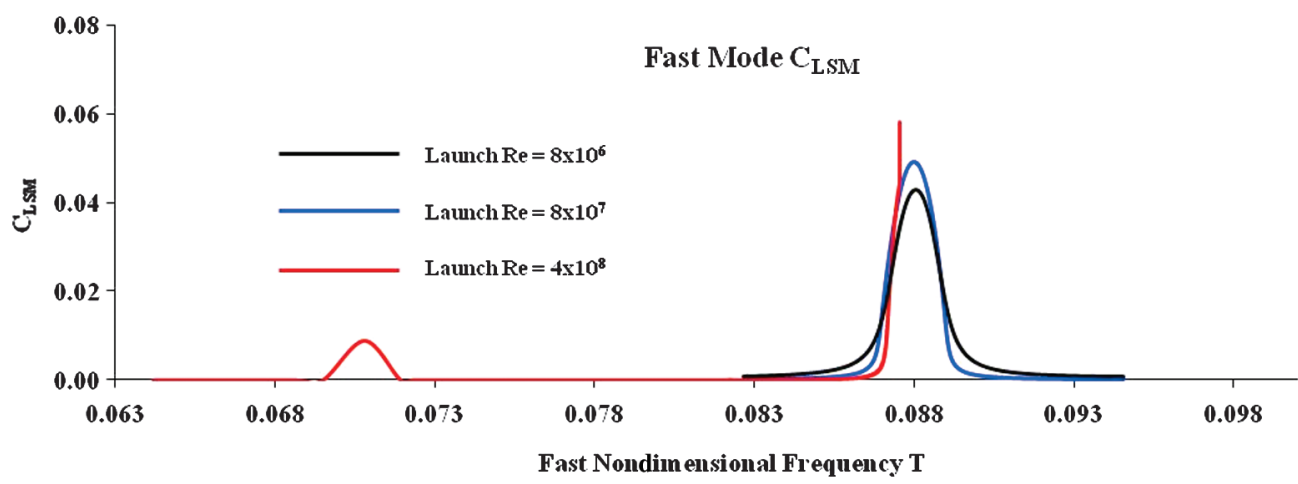

Fig. 9 Comparison of projectile side moment vs payload launch $R e$.

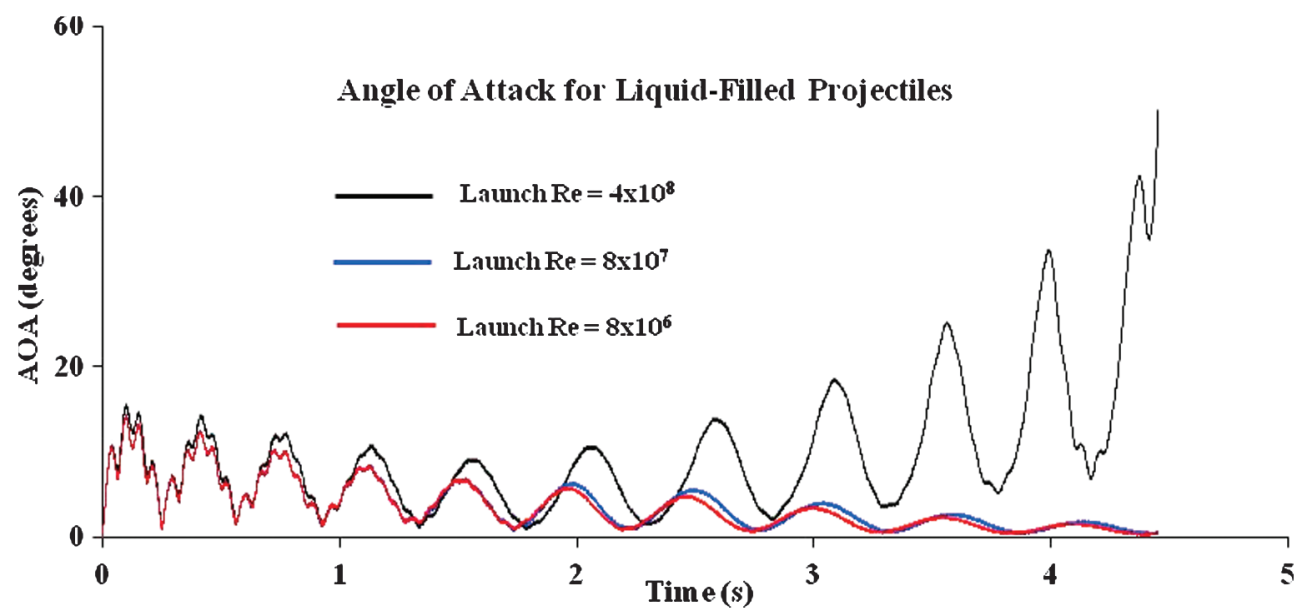

Fig. 10 Projectile AOA vs launch Re of liquid payload. 
Projectile Roll Moment

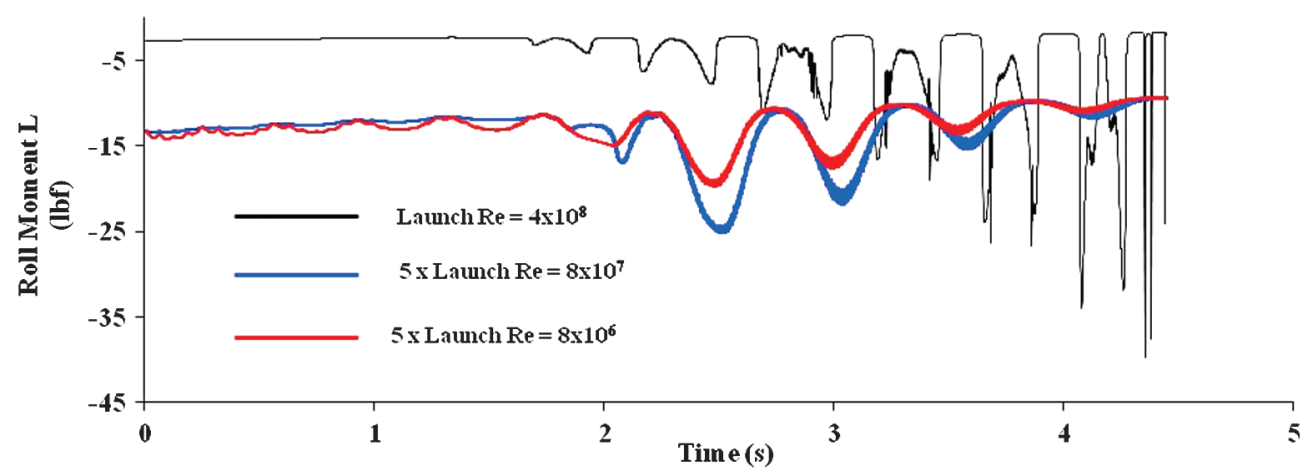

Fig. 11 Comparison of projectile roll moment vs launch $R e$.

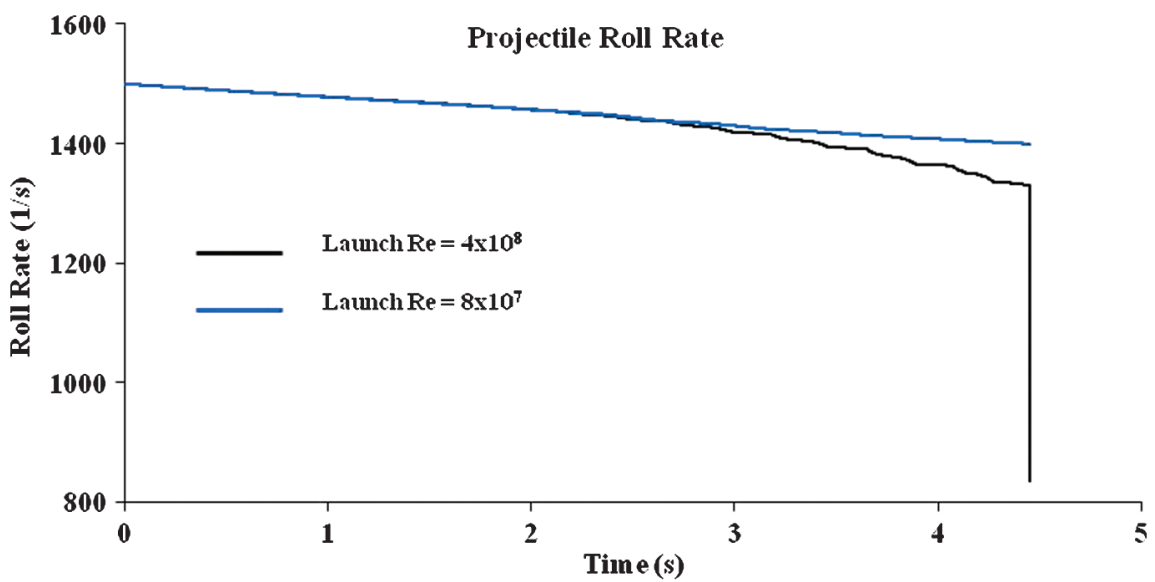

Fig. 12 Comparison of roll rate due to payload launch $R e$.

$R e=4 \times 10^{8}$. This indicates a possible increase in the magnitude of projectile angular motion, which is consistent with the resonance configuration presented in Fig. 4.

Figure 10 compares the AOA of the projectile with liquid payloads having two different launch $R e$.

The projectile with launch $R e=4 \times 10^{8}$ causes the fast frequency at $t \approx 4.5 \mathrm{~s}$ and $T \approx 0.088$ to generate an AOA $>50 \mathrm{deg}$, for which the judgment was make to terminate the numerical integration.

Figure 11 shows the corresponding projectile roll moment $L$ rapidly increasing in magnitude due to the larger Reynolds number. Note that, in the neighborhood of $T=0.088$ and the fast mode, liquid motion is responsible for increasing magnitude of $L$.
Figure 12 shows the corresponding projectile roll rates for the launch $R e=8 \times 10^{6}$ and $4 \times 10^{8}$, which correlates with the size of liquid moment coefficient $C_{L R M}$, as shown in Eq. (15).

Figure 13 compares corresponding pitch rates of the frozen liquid and launch $R e=4 \times 10^{8}$ configurations. The rapid increase in $q$ values near $t \approx 4.5 \mathrm{~s}$ is caused by the fast-mode liquid side moment.

A contrast in projectile yaw rates for frozen and liquid payload is given in Fig. 14. Again, the rapid increase in yaw rate takes place near $t \approx 4.5 \mathrm{~s}$ in the neighborhood of the fast-mode resonance.

These calculations show a significant difference in projectile angular motion when the liquid payload has a launch $R e=4 \times 10^{8}$ compared with the same projectile with a frozen liquid.

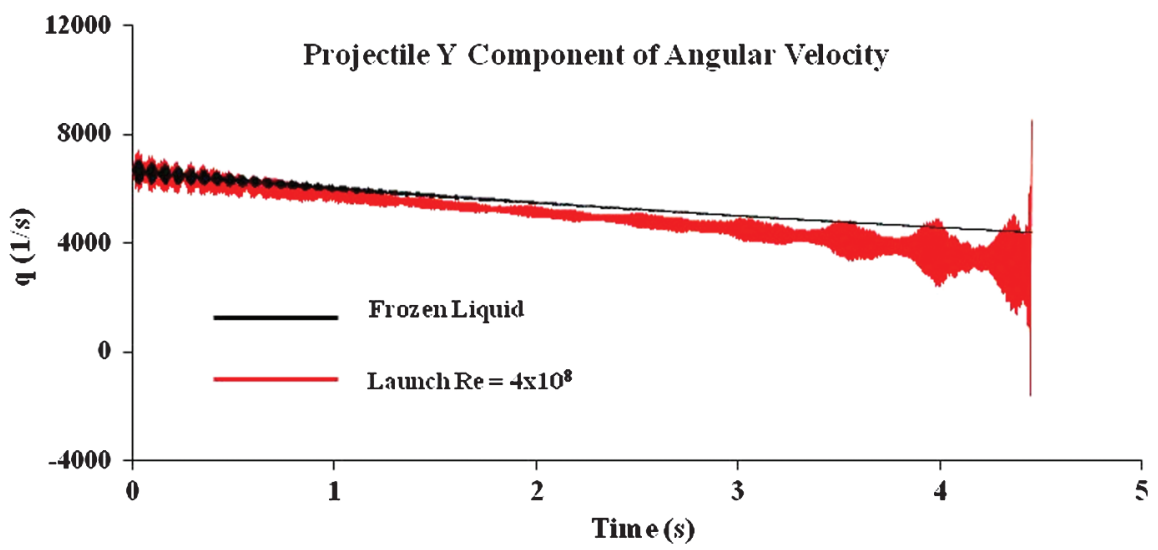

Fig. 13 Comparison of pitch rates due to frozen and liquid payloads. 


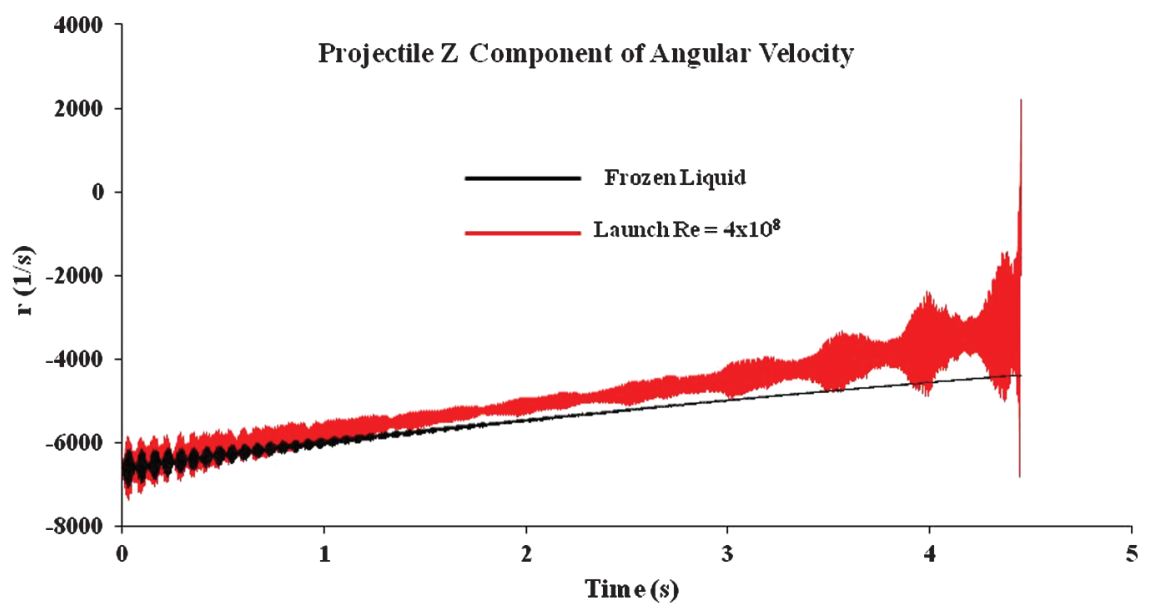

Fig. 14 Comparison of yaw rates due to frozen and liquid payloads.

\section{Conclusions}

An integrated trajectory simulation for a projectile with an internal liquid payload has been created and exercised. The liquid moments are calculated using linear liquid theory applied to a low-viscosity liquid undergoing two-mode steady-state coning motion. High Reynolds number boundary layers approximate the liquid shear moment yielding an average quasi-static liquid moment that is applied to a nonlinear 6-DOF time-dependent trajectory model. These quasi-static averages yield approximate predictions describing the motion of projectiles with low-viscosity liquid payloads filling a cylindrical cavity. The nonlinear 6-DOF motion of a projectile is often well approximated as the sum of fast and slow coning motions. Thus, under these conditions, the linear liquid analysis can predict when a projectile exhibits flight instability due to a liquid payload by tracking the coning frequencies during the 6-DOF numerical integration process. Calculations presented in this paper show how both stable and unstable liquid payload configurations influence trajectory flight parameters. Unstable liquid payload configurations result from an improper combination of liquid Reynolds numbers, payload geometry, and quasi-static projectile coning mode rates. In such cases, the projectile roll rate and axial moment decrease rapidly due to the quasi-static liquid moment.

\section{References}

[1] D'Amico, W. P., and Mark, A., "The Application of a Highly Permeable Medium to Reduce Spin-Up Time and to Stabilize a Liquid-Filled Shell,” U.S. Army Ballistic Research Lab. Rept. MR-02851, Aberdeen Proving Ground, MD, July 1978.

[2] D'Amico, and Clay, W. H., "Flight Tests for Prototype Felt Wedge/ White Phosphorous Improved Smoke Concept," U.S. Army Ballistic Research Lab. Rept. MR-02824, Aberdeen Proving Ground, MD, April 1978.

[3] Cooper, G. R., "Moment Exerted on a Coning Projectile by a Spinning Liquid in a Cylindrical Cavity Containing a Porous Medium," U.S. Army Ballistic Research Lab. Rept. MR-3677, Aberdeen Proving Ground, MD, June, 1988.

[4] Cooper, G. R., "Spinning Projectile with an Inviscid Liquid Payload Impregnating Porous Media," AIAA Journal, Vol. 46, No. 3, March 2008, pp. 783-787. doi: $10.2514 / 1.30481$

[5] Cooper, G. R., "Moments on a Coning M864 by a Liquid Payload: The Candlestick Problem and Porous Media," U.S. Army Research Lab. TR 3837, Aberdeen Proving Ground, MD, July 2006.

[6] Dean, C., Weber, D., Molnar, J., Hollis, M., D'Amico, W., Brandon, F., and Davis, B., "Savage Medical Resupply Projectile," U.S. Army Armament Munitions, Aberdeen Proving Ground, MD, Oct. 1996, http://Handle.dtic.mil/100.2/ADA332808 [retrieved 2011].

[7] Stewartson, K., "On the Stability of a Spinning Top Containing Liquid," Journal of Fluid Mechanics, Vol. 5, No. 4, 1959, pp. 577-592. doi:10.1017/S0022112059000404

[8] Karpov, B. G., "Experimental Observation of the Dynamic Behavior of Liquid Filled Shell," U.S. Army Ballistic Research Lab. Rept. 1171,
Aberdeen Proving Ground, MD, Aug. 1961.

[9] Mark, A., and Mermagen, W. H., "Measurement of Spin Decay and Instability of Liquid-Filled Projectiles via Telemetry," U.S. Army Ballistic Research Lab. Memo.Rept. 2333, Aberdeen Proving Ground, MD, Oct. 1973.

[10] Kitchens, C. W., Jr., Gerber, N., and Sedney, R., "Spin Decay of LiquidFilled Projectiles," Journal of Spacecraft and Rockets, Vol. 15, No. 6, Nov.-Dec. 1978, pp. 348-354. doi: $10.2514 / 3.57326$

[11] Sedney, R., "A Survey of the Fluid Dynamic Aspects of Liquid-Filled Projectiles," AIAA 12th AFM, Snowmass, CO, AIAA Paper 19851822, Aug. 1985.

[12] Wedemeyer, E. H., "Viscous Corrections to Stewartson's Stability Criterion," U.S. Army Ballistic Research Lab. Rept. 1325, Aberdeen Proving Ground, MD, June 1966

[13] Kitchens, C. W., Jr., Gerber, N., and Sedney, R., "Oscillations of a Liquid in a Rotating Cylinder: Part I. Solid-Body Rotation," U.S. Army Ballistic Research Lab. TR 02081, Aberdeen Proving Ground, MD, June 1978

[14] Frasier, J. T., and Scott, W. E., "Stability of a Liquid-Filled Gyroscope: Inviscid Analysis, Viscous Correction, and Experiments," Journal of Spacecraft and Rockets, Vol. 8, No. 5, May 1971, pp. 523-526. doi: $10.2514 / 3.30302$

[15] Weber, D. J., "Simplified Method for Evaluating the Flight Stability of Liquid-Filled Projectiles," Journal of Spacecraft and Rockets, Vol. 31, No. 1, 1994, pp. 130-134. doi: $10.2514 / 3.26412$

[16] Thorwald, H., "Flight Simulation for Liquid-Filled Projectiles," 1987 Scientific Conference on Chemical Defense Research, U.S. Army Chemical Research Development and Engineering Center SP 88013 June 1988, Research Triangle Park, NC, pp. 378-385.

[17] Vaughn, H. R., Wolfe, W. P., and Oberkampf, W. L., "Flight Trajectory Simulation of Fluid Payload Projectiles," Journal of Guidance, Control, and Dynamics, Vol. 9, No. 2, March-April 1986, pp. 213-220. doi: $10.2514 / 3.20092$

[18] Slegers, N., Kyle, J., and Costello, M., "Nonlinear Model Predictive Control Technique for Unmanned Air Vehicles," Journal of Guidance, Control, and Dynamics, Vol. 29, No. 5, 2006, pp. 1179-1188. doi: $10.2514 / 1.21531$

[19] Murphy, C. H., "Angular Motion of a Spinning Projectile with a Viscous Liquid Payload," U.S. Army Ballistic Research Lab. MR-3194, Aberdeen Proving Ground, MD, Aug. 1982; also Journal of Guidance, Control, and Dynamics, Vol. 6, No. 4, July-Aug. 1983, pp. 280-286. doi: $10.2514 / 3.19830$

[20] Murphy, C. H., "A Relation Between Liquid Roll Moment and Liquid Side Moment," Journal of Guidance, Control, and Dynamics, Vol. 8, No. 2, March-April 1985, pp. 287-288. doi: $10.2514 / 3.19977$

[21] Murphy, C. H., Bradley, J. W., and Mermagen, W. H., "Side Moment Exerted by a Spinning Coning Highly Viscous Liquid Payload," U.S. Army Ballistic Research Lab. TR 3074, Aberdeen Proving Ground, MD, Dec. 1989. 